\title{
Development of a Real-Time Stereo Transmission Electron Microscope
}

\author{
H. Tanaka*, T. Kojima*, H. Tsuruta**, J. Chen**, T. Tanji** and M. Ichihashi*** \\ * Department of Electronic Engineering, Nagoya University, Chikusa, Nagoya 464-8603, JAPAN \\ ** Department of Media and Image Technology, Tokyo Institute of Polytechnics, Atsugi, Kanagawa \\ 243-0297, JAPAN \\ *** EcoTopia Science Institute, Nagoya University, Chikusa, Nagoya 464-8603, JAPAN
}

Obtaining three-dimensional (3-D) information with a transmission electron microscope (TEM) is valuable in biology and material science. Stereoscopy allows 3-D information to be taken from two projected images of a specimen that is tilted (stereo pair). To date, 3-D observations have been applied only to stable specimens because the speed of image recording is restricted by the tilting mechanism of the specimen stage. In stereoscopy, the time to record stereo pairs can be greatly reduced by tilting the incident electron beam instead of the specimen stage. Although some reports[1,2] have proposed TEM stereoscopy with tilting illumination, real-time stereoscopy has yet to be realized. Real-time 3-D observation would offer a novel insightful technique for specimen characterization. We have developed a real-time stereo TEM that enables us to directly observe a time series of stereo pairs at the NTSC video-rate of $1 / 30 \mathrm{~s}$.

A schematic diagram of the developed system is shown in FIG. 1. This system has been developed using a $200 \mathrm{kV}$ TEM (Hitachi HF-2000). An electrostatic deflector (A) was placed above the condenser lenses. The incident angle is switched by reversing the polarity of the deflector voltage in synchronization with a vertical synchronizing signal (VSYNC) included in the NTSC signal, that is, the specimen is illuminated obliquely from the left in odd fields while from the right in even fields. Maximum incident angles of up to $\pm 2.3^{\circ}$ are obtained. Another electrostatic deflector (B) was used to correct the image separation between left and right images caused by defocusing of the tilted ray. The lateral resolution of less than $1 \mathrm{~nm}$ could be achieved at a proper underfocus condition without image separation. The depth resolution is $13 \mathrm{~nm}$ at this condition. Each frame records a stereo pair as shown in FIG. 2. A time series of stereo pairs is then transferred to a 3-D display, enabling observation of the 3-D images without special glasses. For a moving specimen, the maximum observable horizontal velocity was estimated to be $8.3 \mathrm{~nm} / \mathrm{s}$ at a magnification of $\times 10000$.

Using the system, we observed $\mathrm{ZnO}$ particles and dislocations in an $\mathrm{Al}$ thin film. FIG. 3(a) shows a stereo pair of the $\mathrm{ZnO}$ particles recorded on one frame with incident angles of $\pm 2.3^{\circ}$. In the enlarged image (FIG. 3(b)), it is obvious that the different images are recorded in alternate fields. FIG. 3(c) shows the stereo pair separated from FIG. 3(a), giving a strong stereo impression with the cross-eye method. Notably, the 3-D images remained clear even while the specimen was moved. FIG. 4 shows a time series of stereo pairs showing dislocations in an Al thin film taken using a double-hole objective aperture. The same dislocations can be seen in the right and left images. We succeeded in observing dislocations in real time while moving the specimen. We are hopeful that this system can give new characterization techniques in crystal material science and physiology.

\section{References}

[1] J. B. Pawley, Proc. 7th EUREM, (Vol. 4) (1980) 58

[2] G. Y. Fan and Mark H. Ellisman, Ultramicrosc., 55 (1994) 155 
[3] This work was supported by Grant-in-Aid for Scientific Research 14205008. We would like to thank Prof. S. Muto and Dr. S. Arai of Nagoya University for preparing the specimens.

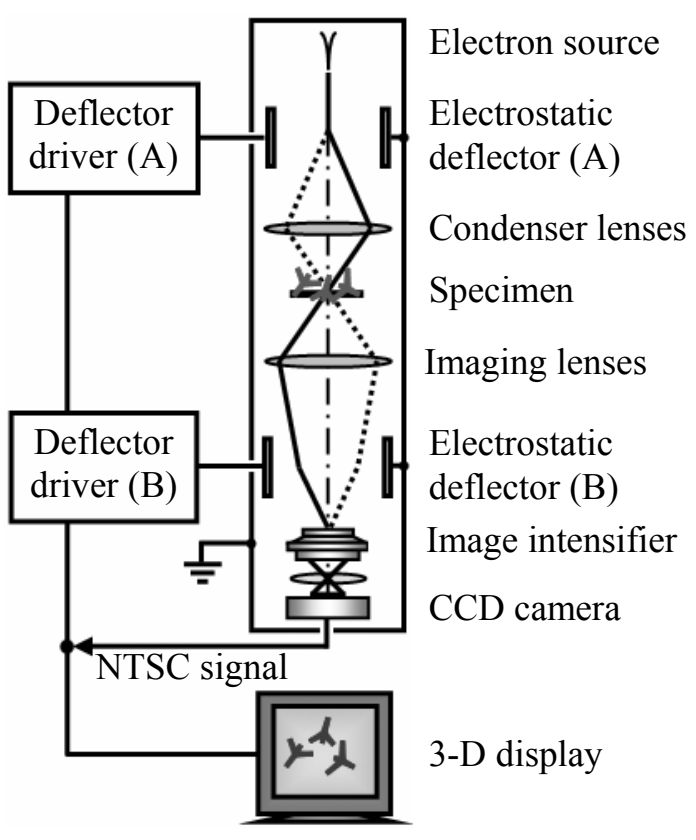

FIG. 1. A schematic diagram of the real-time stereo TEM.

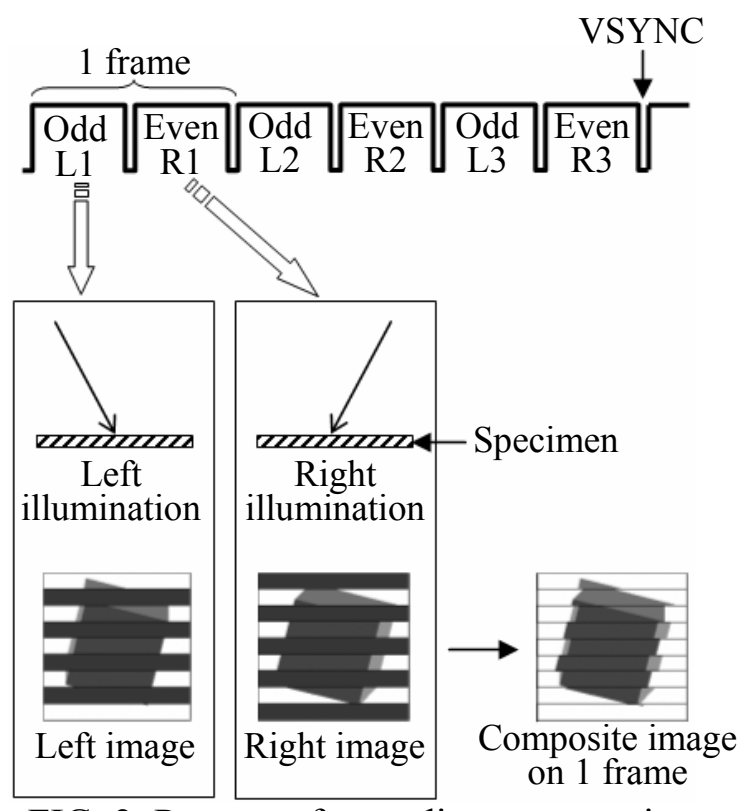

FIG. 2. Process of recording stereo pairs.

FIG. 4. A time series of stereo pairs showing dislocations in an $\mathrm{Al}$ thin film. (seen with cross-eye method.)

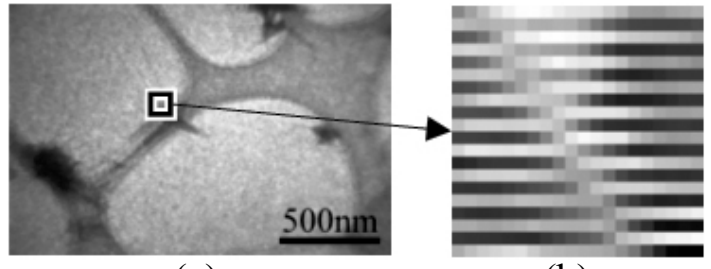

(a)

(b)

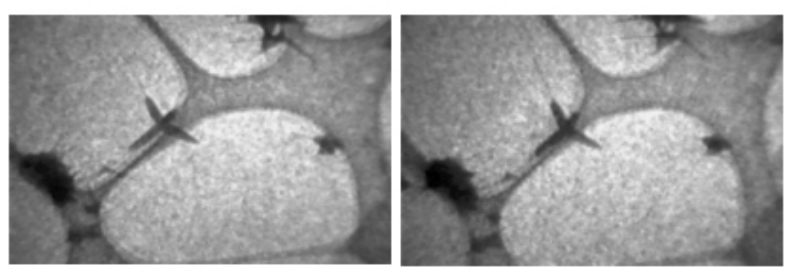

(c)

FIG. 3. (a) Composite stereo pair obtained for $\mathrm{ZnO}$ particles. (b) Enlargement of enclosed area. (c) Stereo pair separated from FIG. 3(a). (seen with cross-eye method.)
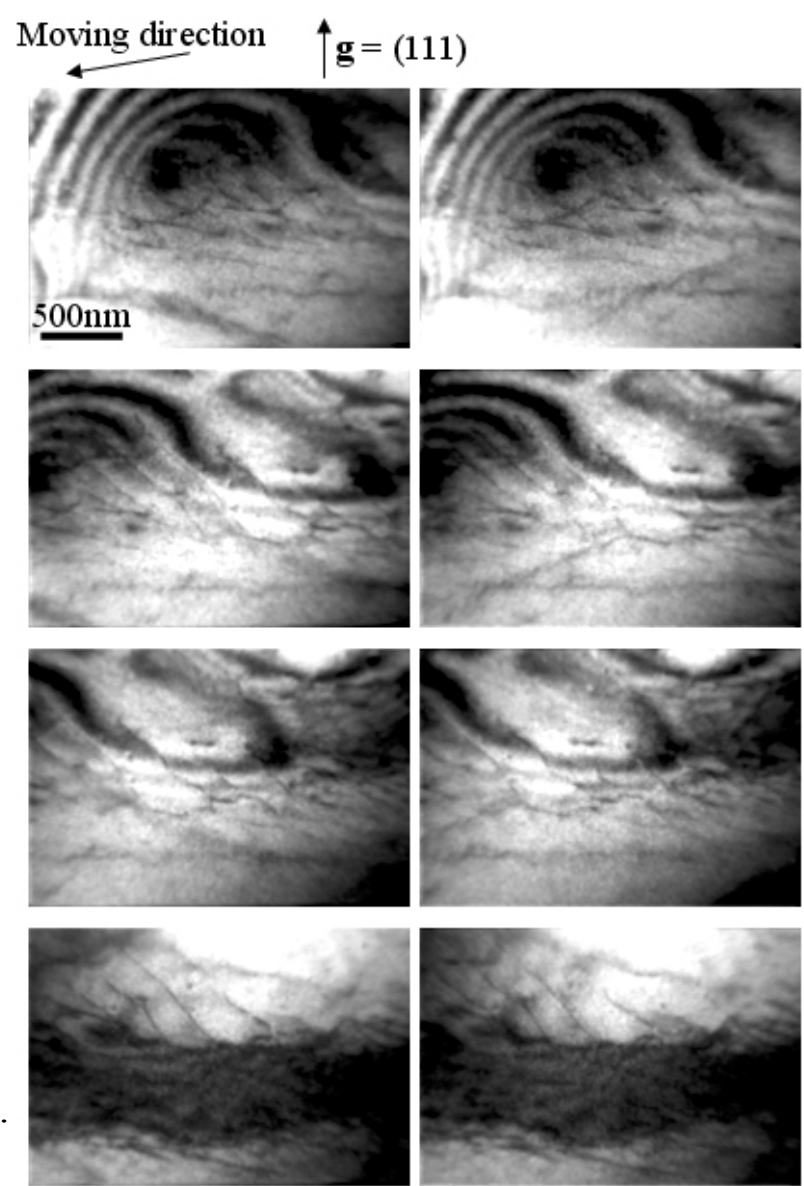